DOI:10.14331/ijfps.2013.330058

\title{
Gravity, Metaphysics or Physics?
}

\author{
Alfonso Leon Guillen Gomez \\ Independent scientific researcher, 127A Street 53A68 Flat 514, Bogota, Colombia
}

Email: aguillen@gmx.net

(Received Sep 2013; Published Dec 2013)

\begin{abstract}
Gravity is the foundation of the current physical paradigm. Due to that gravity is strongly linked to the curvature of space-time, we research that it lacks of a valid physical concept of space-time, nevertheless that from the science philosophy, via substantivalism, it has tried respond. We found that is due to that the gnoseological process applied from the general relativity, necessarily us leads to metaphysic because ontologically space-time is a metaphysical entity. Thus, we arrive to the super substantivalism that from metaphysics gives an answer on space-time rigorously exact with the vision of Einstein on physics. The result is that matter is nothing since all is space-time, i.e. geometry, therefore is a imperative of the physical science break the current paradigm.
\end{abstract}

Keywords: Gravity, Space-time, Metaphysics, Paradigm,

DOI:10.14331/ijfps.2013.330058

\section{INTRODUCTION}

With the formulation of the general relativity $(G R)$ is accepted that it has a scientific physics theory on gravity. Furthermore, due to the numerous experiments made, especially after 1960, all with results impressively accurate to favour of this theory, to its conceptual integration with its antecedent the special relativity $(S R)$ and the numerous theories derived in physics, astronomy and cosmology truly the relativity is the current physics paradigm. But, as the theories of relativity show how motion and gravity are deeply connected with the fundamental nature of space and time (Hawley, 2009), it must give an answer physically consistent on: What is space-time? The problem is that the relativity theory (special relativity and general relativity) operationally defined the space-time, equal that Newton respect to motion and gravity. And like in the case of Newton is the philosophy that enters to define space-time, and in last instance it returns to the old discussion with Leibnitz, taking it out of the sand of physics and putting it into the arena of philosophy.
$S R$ threatens our ordinary distinctions between past, present and future, whilst GR suggests that space and time are not just the neutral stage upon which events take place, but are themselves actors in the drama. Questions about space and time, and about the persistence and motion of material objects have always been central to metaphysics; many of the great philosophers-Aristotle, Descartes and Leibniz for example-contributed significantly to what we now think of as the science of physics, whilst some of the greatest physicistsincluding Newton and Einstein's thought deeply and philosophically about the metaphysical nature of space, time, force and motion. This is a realm in which it can be difficult to draw a sharp distinction between metaphysics and physics (Hawley, 2009).

The physics as the science of matter and energy and their interactions is absent of resolve the nature of the space-time. And in the philosophical and metaphysic theory of the super substantivalism that defines space-time consistently with $G R$, the matter it become nothing. Thus physics loses its main object of study, by this here we formulate that it breaks with 
the current paradigm or it accepts the metaphysics in substitution of the physics science.

\section{GRAVITATIONAL FIELD IS A GEOMETRIC FIELD}

In the general relativity, gravitational phenomena are not caused by gravitational forces but are a manifestation of the non-Euclidean geometry of space-time. The gravitational field is at best a geometric not a physical field(Minkowski, 2012). The curvature of space-time is the gravitational field. Space-time is mathematically defined, like a mathematical model that, of a physical dynamic system, combines space and time into a manifold of four dimensions. Space is the three dimensional continuous, that by coordinates $x_{1}, x_{2}, x_{3}$, geometrically represents the place occupied by universal set of bodies (substantiality model) or constructed by universal set of relations between bodies (relationist model), in geometric terms, bodies have relative position, direction and sense. Time is the one dimension, that by coordinate $\mathrm{x}_{4}$ geometrically represents the instant in that the events occur, placed in time as order of succession, according substantialist model or non distinct from things existing in time, according relationist model, in geometric terms, events has relative order of past, present and future. Thus, in the substantialist model: All things are placed in time as to order of succession; and in space as to order of situation(Newton, 1985). Or in the relationist model: Space to be something merely relative, as time is. Space is an order of coexistences, as time is an order of successions (Leibniz \& Clarke, 1956).

General relativity has a mathematical definition but it lacks of a physical definition of space-time, whereby the philosophy of science replaces to the science of physics, through mainly two conceptions on space-time that are defined in the philosophical theories of substantivalism and relationalism. Both theories recognize two ontological entities: matter and space-time. Matter is compound by particles and its fields. Space-time is a geometric object compound by manifold and metric.

These theories are agreed on the concept of matter. But, these theories differ in that in substantivalism space-time is a geometric real object, its substantial nature is understood as real presence, existing by itself, although, is not material presence, accordingly space-time is an inmmaterial entity. While, in relationalism space-time is a geometric ideal object that has like reference the material existence, exactly to geometric relations between its compounds, accordingly space-time is a category of the thinking.

In general relativity, in substantivalism properties of the metric are intrinsic properties of the manifold, while, in relationalism space-time geometry is a manifestation of a particular field, the gravitational field. I.e., in the substantivalism the gravitational field is nothing, but a local distortion of space-time geometry, while in the relationalism the space-time geometry is nothing since is a simple manifestation of the gravitational field (Rovelli, 1997). It is not clear whether one should identify space-time with the bare manifold or with the metric field(Dorato, 2000). If it is conserved the Einstein's concept that gravitational field is the curvature of space-time then, in both philosophical theories the gravitational field is nothing, since in substantivalism the gravitational field is a property of an immaterial entity and in relationalism the gravitational field is a property of a category of the thinking.

In the theory of general relativity, the mathematical model of a possible universe is the result of any solution to the ten covariant field equations of Einstein, that have the objectives of express, independently of the observer, the general laws of nature, and of describe the static gravitational field resulting of space-time curved by matter. We denote everything but the gravitational field as matter. Our use the word therefore includes not only matter in the ordinary sense, but the electromagnetic field as well (Basri, 1965). In the General Relativity $(G R)$ the distribution of mass-energy $\left(T_{\mu v}\right)$ of the universe determines the geometry of the space-time, according to Einstein's manifold $\left(M_{4}\right)$, Lorentz' metric $\left(g_{\mu v}\right)$; intrinsically curved by the tension that exerts massenergy on $\left(M_{4}\right)$. The expression more compact of Einstein's field equations is:

$$
G_{\mu v}+g_{\mu v} \Lambda=8 \pi T_{\mu v}
$$

where $G_{\mu v}$ is Einstein tensor formed from the second derivatives of the metric tensor that describes space-time curvature and $\Lambda$ is the cosmological constant.

These equations mean: in the case of the substantialist model of space-time that matter curves immaterial spacetime, that plays a dynamic role, as physicist usually say that gravity is an effect of space-time curvature: in Wheeler's expressive words, matter tells space-time how to bend, spacetime tells matter how to curve (Dorato, 2000), additionally $\Lambda$ manifests itself as a constant, repulsive gravitational force between all objects, a built-in tendency of the universe to expand, it is an instance of nontrivial causal powers that we ought to ascribe to space-time itself (Baker, 2005). And in the case of the relationist model of space-time the matter determines that the category of the thinking space-time expresses a curve geometry referred to real world and therefore space-time is an abstract object and the geometry of the set of relations between events is curved, time and space are modes by which we think and not conditions in which we live(Sorli, 2010).

A possible universe model is mathematically represented by $\left(M_{4}, g_{\mu v}, T_{\mu v}\right)$. Where here $M_{4}$ is the manifold that captures topology which may be globally a plane, sphere, saddle, torus, etc. It stores the events according substantivalism or is built by events according relationalism. Each event $(e) \in M_{4}$ has an associated tangent space $R_{4} e$. Also $g_{\mu v}$ is the metric tensor that of manifold captures the geometry, through of connections between events (two points) describes the curvature (gravitational field) and via of the affine connection describe the inertial structure at tangent space, consisting of all tangent vectors to the manifold at a specific event (point). Here $T_{\mu v}$ is the energy-momentum tensor which represents the distribution of matter-energy of that universe. Of course the static gravitational field is a geometric field. When gravitational phenomena are adequately modeled by the space-time curvature it is evident 
that the gravitational field is not something physically real that is, it is not a physical entity. It is a geometric field (Petkov, 2013). We have seen how Einstein defined the gravitational field to be identical to the so-called metric tensor $g_{\mu v}$ used by Riemann to describe the geometry of a space. Einstein's minimalist adoption of $g_{\mu v}$ as the embodiment of the gravitational field was significant and has far-reaching ramifications (Odenwald, 2009). This complete reduction to kinematics yields a purely geometric field of gravitation (composed of metric, curvature, geodesic lines) and a theory which is fully consistent with the original guiding principle of equivalence(Dalton, 1996).

\section{GRAVITATIONAL FIELD LACKS OF ENERGY AND MOMENTUM}

The principle of equivalence establishes that inside of an infinitesimal lapse of the space-time, since in it its variation of gravity can be considered null, a inertial system is equivalent to a gravitational system as also an accelerated uniformly system is equivalent to a gravitational system. Einstein considered the simple case of a transformation from an inertial reference frame of special relativity to a reference frame in uniform rectilinear acceleration. In the accelerated frame of reference a homogeneous inertial field arises. Because of the key empirical fact of the equality of inertial and gravitational mass, Einstein was able to identify this field as a gravitational field (Norton, 1993). From the principle of equivalence, the inertial system, the accelerated uniform system and the gravitational system are equivalents, i.e., kinematics produces inertia, acceleration or gravity according the coordinates of a particular observer, therefore inertia, acceleration and gravity are relative kinematics phenomenon's depending of the system of coordinates of the observer, absolutely a accident geometric of the space-time, referring to the configuration geometric of space-time of a particular observer. The change from the inertial system to the gravitational system or vice versa, or from the accelerated system to the gravitational system or vice versa, is a simple question of change of coordinates, i.e., technically, a geometric manipulation of space-time. Einstein generated a novel theory of static gravitational fields. Einstein and Grossmann published the results of their joint research, in 1913, with the title Outline of a generalized theory of relativity and a theory of gravitation. Its central idea involved the introduction of Ricci and Levi-Civita's fundamental form. They started with the invariant interval of Minkowski in differential form

$$
d s^{2}=c^{2} d t^{2}-d x^{2}-d y^{2}-d z^{2}
$$

Where $(x, y, z, t)$ is, the space-time coordinates of an inertial frame of reference in a Minkowski space-time. Transforming to arbitrary coordinates $x_{\mu}$ for $\mu=1, \ldots, 4$, becomes

$$
d s^{2}=g_{\mu v} d x_{\mu} d x_{v}
$$

Where $g_{\mu v}$ are varies with the position. Einstein employed his principle of equivalence to interpret the matrix of quantities $g_{\mu v}$ that had arisen with the introduction of arbitrary coordinates. In the special case the transformation from (3) to (4) is from an inertial coordinate system to a uniformly accelerated coordinate system. In this case coefficients $g_{\mu v}$ reduces to that of (3), except that $c$ now is a function of the coordinates $\left(x^{\prime}, y^{\prime}, z^{\prime}\right)$. That is, (4) becomes

$$
d s^{2}=c^{2}\left(x^{\prime}, y^{\prime}, z^{\prime}\right) d t^{\prime 2}-d x^{\prime 2}-d y^{\prime 2}-d z^{\prime 2}
$$

According to the principle of equivalence, the presence of a gravitational field was the only difference between the spacetime of Eq (4) and that of special relativity (2). Therefore Einstein interpreted the coordinate dependent $c$ of Eq (4) as representing a gravitational field and, more generally, the $g_{\mu v}$ of Eq (3) as representing a gravitational field. (Norton, 1993).

From principle of equivalence, inertia and gravity are identical in essence. From this and from the results of the special theory of relativity, it follows necessarily that the symmetric 'fundamental tensor $\left(g_{\mu v}\right)$ determines the metric properties of space, the inertial relations of bodies in it, as well as gravitational effects (Norton, 1993). As the gravitational field is a simple consequence of a new expression of Minkowski coordinates and these a new expression of Cartesian coordinates, the gravitational field does not imply energy-momentum.

However, out of limit, of the infinitesimal lapse, the gravitational field has the full properties of curve geometry, from the Gaussian coordinate system according (4) that is a logical generalization of the Cartesian coordinate system. Thus, the space-time continuum of the general theory of relativity is not a Euclidean continuum. We start off on a consideration of a Galilean domain, i.e. a domain in which there is no gravitational field relative to the Galilean reference-body $K$. The behavior of measuring-rods and clocks with reference to $K$ is known from the special theory of relativity, likewise the behavior of isolated material points; the latter move uniformly and in straight lines. Now let us refer this domain to a random Gauss coordinate system or to a mollusc as reference-body $K_{1}$. Then with respect to $K_{1}$ there is a gravitational field $G$. We learn the behavior of measuring-rods and clocks and also of freely-moving material points with reference to $K_{1}$ simply by mathematical transformation. We interpret this behavior as he behavior of measuring-rods, clocks and material points under the influence of the gravitational field $G$. Here upon we introduce a hypothesis: that the influence of the gravitational field on measuring rods, clocks and freely-moving material points continues to take place according to the same laws, even in the case where the prevailing gravitational field is not derivable from the Galilean special case, simply by means of a transformation of coordinates. According to the general principle of relativity, the space-time continuum cannot be regarded as a Euclidean one. We refer the four dimensional space-time continuums in an arbitrary manner to Gauss coordinates (Einstein, 1920).

The intrinsic geometry of curved space-time $g_{\mu v}$, as it has not a constant metric tensor (while the Euclidean space-time, whether) between two giving events, the shortest curve is a 
geodesic, that is equivalent to the straight line of a Euclidean coordinate system. The geodesic is defined as the curve such that a point moving along the curve with the velocity of constant magnitude (i.e. the velocity can change its direction but not its magnitude) has the acceleration vector perpendicular to the given surface, i.e. the acceleration component tangent to the given surface is zero (Pokorny, 2012). General relativity incorporates a number of basic principles that correlate space-time structure with physical objects and processes. Among them is the Geodesic Principle: Free massive point particles traverse timelike geodesics. One can think of it as a relativistic version of Newton's first law of motion (Malament, 2009 ). By the geodesic hypothesis in general relativity, the assumption that the world line of a free particle is a time-like geodesic in space-time is a natural generalization of Newton's first law, that is, a mere extension of Galileo's law of inertia to curved space-time. This means that in general relativity a particle, whose world line is geodesic, is a free particle which moves by inertia (Petkov, 2013).

Since the effect on the bodies of the gravitational field is equivalent to inertia, it confirms that the gravitational field lacks of energy-momentum (surely, the energy-momentum of the Einstein's universe model resides in the energymomentum tensor $T_{\mu v}$ ), in consequence, the gravitational field is an abstract geometric object. As it was noted 90 years ago by (Hilbert, 1917), (Einstein, 1918), (Schrödinger, 1918) and Bauer (1918) within Geometrical Gravity approach (General Relativity) there is no tensor characteristics of the energy-momentum for the gravity field (Baryshev, 2008). Although, Einstein and Grossmann, in 1913, emphasized that the gravity field must have an energy-momentum tensor as all other physical fields. However, in the final version of general relativity Einstein rejected this requirement in order to have a generally covariant gravity theory (Baryshev, 2008) and, in 1918, Einstein said; there may very well be gravitational fields without stress and energy density (Baryshev, 2008).

\section{THE PHYSICAL EFFECT OF THE GEOMETRIC GRAVITATIONAL FIELD}

According the general relativity the physical effect of the geometric gravitational field is kinematics on the matterenergy. Thus, the material motion is described by the geodesic equation (Einstein, 1922)

$$
\frac{d^{2} x_{\mu}}{d s^{2}}+\Gamma_{\alpha \beta}^{\mu} \frac{d x_{\alpha}}{d s} \frac{d x_{\beta}}{d s}=0
$$

Indeed, two particles that seem to be subject to gravitational forces in reality move by inertia according to general relativity since their world lines are time-like geodesics in space-time curved by the particles' masses. The acceleration of the particles towards each other is relative and is caused not by gravitational forces, but by geodesic deviation, which relines in curved space-time. In general relativity the planets, for example, are free bodies which move by inertia and as such do not interact in any way with the Sun because inertial motion does not imply any interaction. The planets' world lines are geodesics, which due to the curvature of space-time caused by the Sun's mass are helixes around the world line of the Sun (which means that the planets move by inertia while orbiting the Sun). Therefore, what general relativity itself tells us about the world is that the apparent gravitational interaction is not a physical interaction in a sense that two particles, which appear to interact gravitationally, are free particles since they move by inertia (Petkov, 2013). This inertial motion inside of the geodesics of the curved space-time produces:

The spherical way of the great bodies like natural satellites, planets and stars and, in general, the way of the bodies, due to the geodesic motion that joins the particles. Planets are round because their gravitational field acts as though it originates from the center of the body and pulls everything toward it (Sears, 2003). The celestial mechanic of the movement of the planets around of Sun and of the satellites around of the planets, in general, any gravitational motion, that occurs always inside geodesics.

Deflection of the light, with approximates to the Sun and the gravitational lens, are consequences of the curvature of the space-time around of the great masses. In 1976, Formalont and Sramek measured with 1 percent accuracy the bending of radio waves emitted from a quasar as it approached eclipse by the Sun (Bruckman \& Esteban, 1993). Also, bending of light ray passing near a massive elliptical galaxy or star, between spherical and elliptical shape objects, which led to appear a small but not negligible coefficient as oblateness independent of mass but depend on shape and geometry of object (Nikouravan B \& Rawal J, 2013).

The gravitational time dilation has inverse relation with gravitational potential (altitude respect to the center of the source of a gravitational field). The lapse measured by a clock is lower respect to a greater potential and potential greater inversely to the distance respect to center of the mass that generates it. The gravitational time dilation has been confirmed by the Gravitational redshift measured first time in the Pound-Rebka experiment.

Gravitational redshift of light produced by the gravitational dilation of time on the electromagnetic waves, that is the reduction of its frequency when they travel between a gravitational greater potential to a lesser potential, at the contrary case due to increase of the frequency, it produces the gravitational blueshift. The frequency or wavelength shift between two identical frequency standards (clocks) placed at rest at different heights in a static gravitational field. The first successful, high-precision redshift measurement was the series of Pound-Rebka-Snider experiments of 1960-1965. Until 2006, the most precise standard redshift test to date was the Vessot-Levine rocket experiment that took place in June 1976 at $10^{-4}$ level. The gravitational redshift could be improved to the $10^{-10}$ level using an array of laser cooled atomic clocks on board a spacecraft which would travel to within four solar radius of the Sun (Will, 2005). In 2012, this test has been improved to the $10^{-6}$ level (Guéna et al., 2012). The Shapiro time delay that is directs to the curvature of a given space-time, due to that the waves and particles travel a path more long and they use a greater time. This delay is measured respect to time used in a flat space-time, i.e., Minkowskian. Using the Viking landers on Mart yielded an 
agreement with Einstein's theory with a impressive accuracy of 0.1 percent (Bruckman \& Esteban, 1993).

The geodetic precession is produced in general in the spinning bodies in free fallen and in particular in the planets and is described as the very slow change in the direction of its rotational axis. In the long time, it traces, with respect to south and north poles, two cones (26.000 years in the case of Earth). In absence of the geodetic precession, planets would repeat the same orbit, i.e, same geodesy, but as this is curve the rotational axis after it has completed one orbit, its direction differs ever so slightly from the way it started, this is a property of the parallel transport at a curve ("Einstein online," 2013). In the solar system, a part of precession of the orbit of a planet is caused by the gravity of the others planets (combined effects of the gravitational fields of the planets in the curvature of the global space-time) and the rest, called anomalous precession of the perihelion of the orbits of the planets, by the curvature of the space-time caused by sun. The curvature of space changes the way that our planet's spin axis processes-these changes are very small and hard to measure (NASA, 2000), since in a flat space-time the direction of the rotational axis is constant in the time. In 2011, the geodetic precession, according Einstein, was confirmed at an accuracy of $0.28 \%$, in the GP-B prove (Everitt et al., 2011). The space-time vortex is produced by the twisted of space-time around of a planet due to its rotation. In general is called the interior gravitomagnetism the space-time-dragging by the rotation of a planet. Also, it produces the exterior gravitomagnetism that is the spacetime-dragging by the orbital motion of a planet. In 2011, the interior gravitomagnetism, according Einstein, was confirmed at an accuracy of $19 \%$, in the GP-B prove (Everitt et al., 2011).

The gravitational waves, that are the transversal propagation of undulations of the curvature of space-time, are caused by asymmetric accelerations of binary pulsars or systems compound of a pair of white dwarfs, neutron stars, black holes etc., also, in the gravitational asymmetric collapses, or asymmetric explosions in the nuclei of Galaxies, or asymmetric outbreaks of supernovas, or in the asymmetric sprouting of a stellar system or at the moment of the Big Bang, in that case called fundamental gravitational waves. In the systems of two masses, with time occurs a variation of the common centre of masses of system, this produces a quadruple that reunites the relation of the energy of four nonsymmetrical angular moments of two masses. The gravitational waves carry energy, which in the lowest order is proportional to the quadruple moment of the distribution of the mass-energy, in particular, of system of two masses. This radiation is originated in lost of the energy kinetic and/or potential, during the no uniform accelerations of the two masses or, in general, in the asymmetric accelerations. Thus, during the gravitational radiation, the mass in rest of the particles, constitutive of mass, does not change. Neither, the curvature of space-time is source of energy carried by the gravitational waves. They deform the geometry of the spacetime, still more, and therefore, they produce more gravity, since they are nonlinear waves. The gravitational waves cause that the distances between the particles are changing over time. Sometimes, the gravitational wave stretches all vertical distances between particles and, at the same time, squeezes all horizontal distances. At other times, all horizontal distances are stretched while all vertical distances are squeezed (online, 2013). On the existence of the gravitational waves, exist the binary pulsar B1913+16 that was the first to be discovered by (Hulse \& Taylor, 1975). The measured rate of change of orbital period agrees with that expected from the emission of gravitational radiation, according to general relativity to within about 0.2 percent (Weisberg \& Taylor, 2005).

\section{SPACE-TIME LIKE AN IMMATERIAL REAL ENTITY}

Therefore, the gravitational phenomenon includes two classes of interactions between the matter and the space-time: The matter acts on the space-time curving it, twisting it, dragging it and dynamically wavy its curvature. The spacetime acts on matter giving it form, causing it moves within geodesics and producing the effects of the gravitational lens, the dilation of time, the gravitational redshift, the Shapiro time delay and the geodetic precession. Both classes of interactions are supported in experiments. Thus, the spacetime has a real presence, like passive object, or like active subject with relation to matter. Therefore, surely the philosophical conception that results consistent with the space-time like a real entity is the conception of the substantivalism. It is currently the most plausible theory of space-time consistent with the general theory of relativity (Grant, 2013). In the aftermath of the rediscovery of Einstein's hole argument by Earman and Norton (1987), we hear that the ontological relational/substantival debate over the status of space-time seems to have reached stable grounds. Despite Einstein's early intention to cast GR's space-time as a relational entity to the Leibniz-Mach, most philosophers of science feel comfortable with the now standard sophisticated substantivalist (SS) account of spacetime. Furthermore, most philosophers share the impression that although relational accounts of certain highly restricted models of GR are viable, at a deep down level, they require substantival space-time structures. SS claims that although manifold space-time points do not enjoy the sort of robust existence provided by primitive identity, it is still natural to be realistic about the existence of space-time as an independent entity in its own right. It is argued that since the bare manifold lacks the basic space-time structures such as geometry and inertia-one should count as an independent space-time the couple manifold + metric $(M, g)$. The metric tensor field of $G R$ encodes inertial and metrical structure so, in a way, it plays the explanatory role that Newtonian absolute space played in classical dynamics. In a nutshell, according to the SS account of space-time, one should view the metric field of GR as the modern version of a realistically constructed space-time since it has the properties-or contains the structures-that Newtonian space had (Cala Vitery, 2006). However, although it seems solved the philosophical question about space-time, physically the space-time is nothing since considered in itself or as a gravitational field lacks of energymomentum. But, according to SS, all of the explanatory work 
is done by the presence of the metric fields in space-time and not by the manifold. It is the metric field tensor, for example, that is appealed to in explaining the motion of material things, physical phenomena such as free fall, acceleration and light propagation, and the distinction between spatial and temporal directions (Hoefer, 1996). They argue, that since the metric field is dynamic, and thus carries both energy and momentum as a result of the presence of the gravitational field, that it ought to count along with other physical fields as matter rather than as space-time. Otherwise, the distinction between space-time and matter becomes obscured (Grant, 2013). Of course, SS tends to unify the model $\left(M_{4}, g_{\mu v}, T_{\mu v}\right)$ like an only expression of matter, since the model $S S$ consists in $\left(M_{4} \cup g_{\mu v}, T_{\mu v}\right)$ and as $g$ is dynamic then apparently the model only represents matter. That the metric field is dynamic is questionable since it is not by itself but the effect of asymmetrical movements of matter that provides the energy and it generates the undulation of the curvature. Such energy is kinetic-potential energy transformed from the asymmetric motion of matter, which it becomes undulation of the curvature. The resulting gravitational wave, although this disturbance comes from the curvature of space-time, belongs undoubtedly to the matter, and thus the gravitational wave is a component of the energy-momentum tensor according to the model of general relativity. It is noted that, Einstein's equation does not express the energy conservation for matter plus gravity field (Baryshev, 2008). In general relativity there are no fundamental laws of conservation of energymomentum and angular momentum of matter and gravitational field taken together (Logunov \& Mestvirishvili, 1989). Thus, space-time curvature alone cannot initiate motion Van Vlandern (Tom, 2004). Therefore, if space-time is an immaterial entity then, as can the space-time be a subject active on the matter? Or as can the space-time be an object passive from matter? These questions are not for that respond the science of the physics, but for that respond the philosophy of the science or metaphysics. As the philosophy of the science, through of substantivalism, cannot give a consistent answer then metaphysics must give it. The profound reason is that in the substantivalism the space-time like an immaterial entity ontologically corresponds to the metaphysics. Space-time substantivalism is a metaphysical theory. It is the thesis that space-time is something real: it is a robust entity with determinate properties which can exist independently of the material objects and events which may occupy it (Grant, 2013). The metaphysics via super substantivalism affirms that only exist space-time since the properties we typically conceive of as belonging to ordinary objects and events are direct properties of the space-time manifold. Hence, when we talk about an object or an event as being identified with a region of space-time, all we really mean is that the matter represented by $T$ are properties of $M$ (or a collection of manifold points) matter is reduced to the geometrical properties of the metric field, so that all of the

\section{REFERENCES}

Baker, D. J. (2005). Spacetime substantivalism and Einstein's cosmological constant. Philosophy of science, 72(5), 12991311 . matter in $T$ is fully incorporated into $g$, and there is no independent $T$ left. This is the theory of geometrodynamics. The identification of matter with space-time therefore involves reducing each material thing in the world (each elementary particle, each light ray etc...) to $g$. On this view, matter is literally constituted of space-time, where space-time is represented by $M$ and $g$ and the properties we typically conceive of as belonging to objects and events are properties of space-time insofar as the matter in $\mathrm{T}$ represents space-time in addition to $M$ and $g$. On this view, the relationship between the components of the model $(M, g, T)$ is the same as for the manifold substantivalist (matter still occupies the manifold), but the matter in $T$ also represents space-time. What it means to identify matter with space-time is therefore to include matter in our definition of space-time (Grant, 2013). Surely, super substantivalism is rigorously just with the vision of Einstein on the physics.

In the case of the relationalism might exist a dualism between the philosophy of the science and the metaphysics giving an answer on space-time. The philosophy of the science via the relationalism has evaporated the space-time, i.e., it has reduced to nothing. Therefore, it is an unacceptable theory. If the space-time, like a thinking category, is considered ontologically like an abstract entity, then correspond to metaphysics give an answer. The theory of abstract objects is a metaphysical theory. Whereas physics attempts a systematic description of fundamental and complex concrete objects, metaphysics attempts a systematic description of fundamental and complex abstract objects. Abstract objects are the objects that are presupposed by our scientific conceptual framework (Zalta, 1983). However, in the relationalism does not take into account Leibnitz's profound metaphysics of monads. Most discussions of the ontology of space-time theories only consider the phenomenological aspect of Leibniz's argument (Weinert, 2000). In consequence, relationalism has not a metaphysical version.

\section{CONCLUSIONS}

The result, of this work, is that truly no exists a theory on gravity, due to the unacceptable answers from current paradigm, based in general relativity, on space-time, and in summary are as: The general relativity gives a mathematical definition but it lacks of a physical definition. The science philosophy via sophisticated substantivalism, tends reduces space-time to the material phenomena, i.e., space-time is matter, and via relationalism reduces space-time to nothing. Metaphysics from super substantivalism becomes all spacetime and evaporates the material reality, i.e., matter becomes nothing. Therefore, is a scientific imperative that physics responds: What is space-time?. This means that it must break with the current physical paradigm on gravity. The alternative is accepted that physics is an extension of metaphysics.

Baryshev, Y. V. (2008). Energy-momentum of the gravitational field: crucial point for gravitation physics and cosmology. arXiv preprint arXiv:0809.2323. 
Basri, S. A. (1965). Operational foundation of Einstein's general theory of relativity. Reviews of Modern Physics, 37(2), 288.

Bruckman, W., \& Esteban, E. (1993). An alternative calculation of light bending and time delay by a gravitational field. American Journal of Physics, 61, 750.

Cala Vitery, F. (2006). Relational Spacetime Ontology. Paper presented at the Relational Spacetime Ontology. In: UNSPECIFIED.

Dalton, K. (1996). Gravity, geometry, and equivalence. arXiv preprint gr-qc/9601004.

Dorato, M. (2000). Substantivalism, relationism, and structural spacetime realism. Foundations of Physics, 30(10), 1605-1628.

Earman, J., \& Norton, J. (1987). What price spacetime substantivalism? The hole story. British Journal for the Philosophy of Science, 515-525.

Einstein, A. (1918). Prinzipielles zur allgemeinen Relativitätstheorie. Annalen der Physik, 360(4), 241-244.

Einstein, A. (1920). Relativity: The special and the general theory: Penguin. com.

Einstein, A. (1922). The general theory of relativity The Meaning of Relativity (pp. 54-75): Springer.

Einstein online. (2013), from http://www.einsteinonline.info/aboutEO/aboutUs

Everitt, C., DeBra, D., Parkinson, B., Turneaure, J., Conklin, J., Heifetz, M., . . . Kolodziejczak, J. (2011). Gravity Probe B: Final results of a space experiment to test general relativity. Physical Review Letters, 106(22), 221101.

Grant, S. (2013). The Metaphysics of Space-time Substantivalism: A Methodological Study of the Structure of Space-time in the Context of General Relativity. University of Otago.

Guéna, J., Abgrall, M., Rovera, D., Rosenbusch, P., Tobar, M. E., Laurent, P., . . . Bize, S. (2012). Improved Tests of Local Position Invariance Using $\wedge\{87\} \mathrm{Rb}$ and $^{\wedge}\{133\} \mathrm{Cs}$ Fountains. Physical Review Letters, 109(8), 080801.

Hawley. (2009). Metaphysics and Relativity. McGonigal, Routledge.

Hilbert, D. (1917). Die Grundlagen der Physik.(Zweite Mitteilung). Nachrichten von der Gesellschaft der Wissenschaften zu Göttingen, Mathematisch-Physikalische Klasse, 1917, 53-76.

Hoefer, C. (1996). The metaphysics of space-time substantivalism. The Journal of Philosophy, 93(1), 5-27.

Hulse, R. A., \& Taylor, J. H. (1975). Discovery of a pulsar in a binary system. The Astrophysical Journal, 195, L51-L53.
Leibniz, G. W., \& Clarke, S. (1956). 1717. The LeibnizClarke Correspondence, Together with Extracts from Newton's "Principia" and "Optics" ed. HG Alexander (Manchester: University of Manchester), 20-21.

Logunov, A. A., \& Mestvirishvili, M. A. (1989). Relativistic theory of gravitation. Moscow Izdatel Nauka, 1 .

Malament, D. (2009 ). On the Status of the Geodesic Principle in General Relativity. philsci-archive.

Minkowski, H. (2012). Space and time.

NASA. (2000). Precession in Curved Space The Geodetic Effect. NASA.

Newton, I. (1985). Mathematical principles of natural philosophy; Optics: Franklin Library.

Nikouravan B, \& Rawal J, J. (2013). Deflection of Light Near Elliptical Galaxies or Stars in General Relativity. Advanced Studies in Theoretical Physics, 7(17-20). doi: doi.org/10.12988/astp.2013.3993

Norton, J. D. (1993). General covariance and the foundations of general relativity: eight decades of dispute. Reports on progress in physics, 56(7), 791.

Odenwald, S. (2009). Space-time: Gravity. Redwood City, CA: Digital Universe Foundation.

Petkov, V. (2013). Can Gravity be Quantized? Institute for Foundational Studies Hermann Minkowski.

Pokorny, P. (2012). Geodesics Revisited. Chaotic Modeling and Simulation.

Rovelli, C. (1997). Half way through the woods, in The Cosmos of Science. University of Pittsburgh Press and University.

Schrödinger, E. (1918). The energy components of the gravitational field. Phys. Z, 19(4).

Sears, D. (2003). Why are planets round? Scientific American.

Sorli, A. S. (2010). Physical time is run of clocks in timeless space. Prespacetime Journal, 1(2), 198-200.

Tom, V. F. (2004). Does Gravity Have Inertia?. Meta Research.

Weinert, F. (2000). Relationism and Relativity. Philosophy of Science, 51, 561-585.

Weisberg, J., \& Taylor, J. (2005). Binary Radio Pulsars (ASP Conf. Ser. 328), ed. FA Rasio \& IH Stairs (San Francisco, CA: ASP), 25.

Will, C. M. (2005). The confrontation between general relativity and experiment. arXiv preprint gr-qc/0510072.

Zalta, E. (1983). Abstract objects: An introduction to axiomatic metaphysics: Springer. 